\title{
Protective effect of Rhizoma Dioscoreae extract against alveolar bone loss in ovariectomized rats via regulation of IL-6/STAT3 signaling
}

\author{
ZHI-GUO ZHANG ${ }^{1}$, YAN-JING CHEN ${ }^{1}$, LI-HUA XIANG ${ }^{1}$, JING-HUA PAN ${ }^{1}$, \\ ZHEN WANG ${ }^{1}$, GARY GUISHAN XIAO ${ }^{2}$ and DA-HONG JU ${ }^{1}$ \\ ${ }^{1}$ Institute of Basic Theory, China Academy of Chinese Medical Sciences, Beijing 100700; \\ ${ }^{2}$ School of Pharmaceutical Science, Dalian University of Technology, Dalian, Liaoning 116024, P.R. China
}

Received February 9, 2017; Accepted September 7, 2017

DOI: 10.3892/ijmm.2017.3130

\begin{abstract}
The aim of the present study was to assess the effectiveness of Rhizoma Dioscoreae extract (RDE) on preventing rat alveolar bone loss induced by ovariectomy (OVX), and to determine the role of interleukin-6 (IL-6)/signal transducer and activator of transcription 3 (STAT3) signaling pathway in this effect. Female Wistar rats were subjected to OVX or sham surgery. The rats that had undergone OVX were treated with RDE (RDE group), vehicle (OVX group) or $17 \beta$-estradiol subcutaneous injection (E2 group). Subsequently, bone metabolic activity was assessed by analyzing 3-D alveolar bone construction, bone mineral density, as well as the plasma biomarkers of bone turnover. The gene expression of alveolar bone in the OVX and RDE groups was evaluated by IL-6/STAT3 signaling pathway polymerase chain reaction (PCR) arrays, and differentially expressed genes were determined through reverse transcription-quantitative PCR. The inhibitory effect of RDE on alveolar bone loss in the OVX group was demonstrated in the study. In comparison with the OVX group, the RDE group exhibited 19 downregulated genes and 1 upregulated gene associated with the IL-6/STAT3 signaling pathway in alveolar bone. Thus, RDE was shown to relieve OVX-induced alveolar bone loss in rats, an effect which was likely associated with decreased abnormal bone remodeling via regulation of the IL-6/STAT3 signaling pathway.
\end{abstract}

Correspondence to: Professor Da-Hong $\mathrm{Ju}$, Institute of Basic Theory, China Academy of Chinese Medical Sciences, 16 Nanxiaojie, Dongzhimennei, Beijing 100700, P.R. China

E-mail: judahong@sohu.com

Professor Gary Guishan Xiao, School of Pharmaceutical Science, Dalian University of Technology, 2 Linggong Road, Ganjingzi, Dalian, Liaoning 116024, P.R. China

E-mail: gxiao@dlut.edu.cn

Key words: alveolar bone loss, interleukin-6/signal transducer and activator of transcription 3 signaling pathway, herbal medicine, ovariectomized rats, Rhizoma Dioscoreae

\section{Introduction}

Osteoporosis is a common postmenopausal disease that markedly affects the quality of life of the patients (1). There is a known association between alveolar bone loss and osteoporosis in women following pausimenia (2). Furthermore, alveolar bone provides essential tooth support through desmodontal fiber anchoring. It was previously reported that alveolar bone mass maybe reduced and alveolar bone structure may be altered in patients suffering from osteoporosis (3). During the early postmenopausal period, alveolar bone loss occurs rapidly, but this process is leveled out in the 6th postmenopausal year, which likely results from postmenopausal estrogen reduction (4). Alveolar bone loss causes tooth loss or mobile teeth, severely compromising postmenopausal quality of life (5).

Estrogen (6), parathyroid hormone (PTH) (7) and bisphosphonates (8) are currently used for preventing postmenopausal alveolar bone loss. However, it was indicated that long-term use of these agents may be associated with side effects, including higher risk of endometrial and ovarian cancer $(9,10)$, nervous system disorders (11), osteonecrosis of the jaws (12) and venous thromboembolism (13). A substitutive method or drug with verified safety and efficiency is urgently required for treating alveolar bone loss. In recent decades, certain herbal medicines or botanical drugs have been widely recognized as effective remedies for relieving or treating alveolar bone loss (14-16).

Rhizoma Dioscoreae (RD), a Chinese medicinal herb/medicinal food, has long been used to promote bone and tooth strength in China (17). Our previous study demonstrated that RD extract (RDE) exerts a protective effect on maintaining alveolar bone among rats subjected to ovariectomy (OVX) through the regulation of $\mathrm{p} 38$ mitogen-activated protein kinase (MAPK) and Wnt signaling pathway (18). However, it has not been fully elucidated whether this effect is associated with other signaling pathways.

Previous studies have demonstrated that the interleukin-6 (IL-6)/signal transducer and activator of transcription 3 (STAT3) signaling pathway in osteoclasts and osteoblasts plays a central role in osseous metabolism and remodeling $(19,20)$. Moreover, using bioinformatics methods in our previous 
study, we predicted that RDE protection against alveolar bone loss may be associated with the IL-6/STAT3 signaling pathway (21). The aim of the present study was to analyze the inhibitory effect of RDE on alveolar bone loss among OVX rats, and investigate the association between this effect and the IL-6/STAT3 signaling pathway.

\section{Materials and methods}

Preparation of $R D E$. RDE was prepared as previously reported (22), and that same batch of extract was used in the present study.

Grouping and treating animals. A total of 48 Wistar female virgin rats (aged 6 months and weighing $310 \pm 20.0 \mathrm{~g}$ ) were acquired from the Experimental Animal Center in the Academy of Military Medical Sciences [SCXK-(Military) 2014-005, Beijing, China]. The protocol involving animals in the present study was authorized by the Institutional Ethics Committee of China Academy of Chinese Medical Sciences (approval no. 2015-009). Sham surgery $(n=12)$ or bilateral OVX $(n=36)$ using a dorsal incision was conducted on the rats following acclimatization. The rats undergoing OVX were divided in three groups based on the treating agent, namely control (OVX), RDE and $17 \beta$-estradiol (E2) groups. Each group contained 12 rats. Subsequently, $17 \beta$-estradiol (Sigma-Aldrich, St. Louis, MO, USA) was dissolved in ethanol and diluted with olive oil. The preparation was used for daily subcutaneous injection in the E2 rats at a dosage of $30 \mu \mathrm{g} / \mathrm{kg}$ body weight. RDE was dissolved with distilled water and force-fed to RDE rats at a dosage of $1.3 \mathrm{~g} / \mathrm{kg}$ body weight/day, which was calculated using the human recommended dosage (30 g/day) proposed by Chinese Pharmacopeia and the weight ratio of rat and human. OVX and sham rats were force-fed equal quantities of distilled water, and standardized rat food was provided to all subjects throughout the study (Animal Center of the Fourth Military Medical University, Xi'an, China). The rats were treated with agents or distilled water for 7 days postoperatively and the treatment was maintained until the 13th week, with body weight monitoring of each subject once weekly.

Preparation of specimens. On the day after the final treatment, xylazine $(12 \mathrm{mg} / \mathrm{kg})$ and ketamine $(80 \mathrm{mg} / \mathrm{kg})$ were intraperitoneally injected to anesthetize the animals, which were subsequently sacrificed by exsanguination. The uteri were excised and immediately weighed (23). The abdominal aorta was punctured prior to death to collect blood specimens into heparinized tubes. Subsequently, the blood specimens were separated by centrifugation at 3,000 $\mathrm{x} \mathrm{g}$ at a temperature of $4^{\circ} \mathrm{C}$ for $10 \mathrm{~min}$, and then aliquoted and preserved at $-80^{\circ} \mathrm{C}$ until use. The left mandibles were excised and preserved at $-80^{\circ} \mathrm{C}$ for reverse transcription-quantitative polymerase chain reaction (RT-qPCR) and microarrays. The right mandibles were excised, immersed into normal saline solution and preserved at $-20^{\circ} \mathrm{C}$, with the aim of measuring bone mineral density (BMD) and studying the microscopic structure using microscopic computed tomography (micro-CT).

Biomarkers of bone turnover. Enzyme-linked immunosorbent assays (ELISA; Sunbio, Inc., Beijing, China) were conducted to assess plasma concentrations of bone formation and bone absorption biomarkers, such as alkaline phosphatase (ALP) and tartrate-resistant acid phosphatase (TRAP), in control, standardized and duplicated experiments. An ELISA reader (Bio-Tek, Colmar, France) was used to read $450 \mathrm{~nm}$ absorption values.

Micro-CT analyses. Untreated right mandibles were scanned with high-resolution micro-CT (SkyScan 1172 micro-CT system; SkyScan, Antwerp, Belgium) which applied cone beam reconstruction for the determination of the cone geometric construction of the X-ray source. The desktop SkyScan micro-CT system was operated as previously described (24). The desirable resolution value of $6.8 \mu \mathrm{m}$ was obtained by placing a specimen on the rotating stage and translating it along the persistently varying magnifying stage. The rotation angle of a specimen was $185^{\circ}$, and an image was generated with the specimen rotating for $0.9^{\circ}$. The repeatability of this protocol was verified by performing repetitive scanning from the start of this experiment. The resulting gray level images were denoised with a low-pass filter, and a constant threshold value was used to determine the trabeculae.

Following image capture $(100 \mathrm{keV}, 100 \mu \mathrm{A})$, a quadrate region of interest (ROI) $(1 \times 1 \mathrm{~mm})$ was constructed using CT analyser, which was affiliated to SkyScan on the sagittal planes of the first molar teeth. None of the selected ROIs overlapped with any tooth roots. As a 3-D rebuilding software affiliated to SkyScan, NRecon was applied for establishing a volume of interest (VOI) with a cubical mass (1x1x1 mm) underneath the bottom of the first molar crown, with a vertical distance of $1.5 \mathrm{~mm}$, and the trabeculae in VOI were measured morphologically by the standardized SkyScan software package. Subsequently, degree of anisotropy (DA), structure model index (SMI), trabecular number (Tb.N), trabecular separation (Tb.Sp), trabecular thickness (Tb.Th), bone volume fraction (BV/TV) and BMD were assessed for a certain VOI using 3-D analysis (25).

$R T$ - $q P C R$ array assay. Alveolar bone from $6 \mathrm{OVX}$ and $6 \mathrm{RDE}$ rats was used for RT-qPCR. The differential expression profiles of IL-6/STAT3 signaling pathway-related genes were analyzed by the rat IL-6/STAT3 signaling pathway PCR array (PARN-160Z; Qiagen, Valencia, CA, USA) obtained from Kangchen Biotech (Shanghai, China). A total of 84 essential genes that may be involved in the activation of the IL-6/STAT3 signaling pathway and downstream reactions were profiled by this PCR array. RNA was extracted and utilized to synthesize First-Strand cDNA by RT2 First Strand kit (Qiagen, Manchester, UK) on the basis of standardized protocol and the cDNA template was mixed with RT2 SYBR-Green qPCR Master mix (Qiagen, Germantown, MD, USA), which was ready to be used in the specific kit. Subsequently, mixed reagent and template was injected into wells on PCR array plates, which contained cytokines and IL-6/STAT3 signaling-associated genes, at a dosage of $25 \mu \mathrm{l}$ for 96 -well plates, to perform RT-qPCR. An instrument-specific software was utilized to calculate quantification cycles $(\mathrm{Cq})$ of all genes throughout each PCR experiment and the $2^{-\Delta \Delta \mathrm{Cq}}$ approach was applied for calculating fold-change in the gene-expressing profiles for comparing between any two means. 
Table I. Primers used for RT-qPCR analysis.

\begin{tabular}{|c|c|}
\hline Transcript & Sequence (5'-3') \\
\hline Gapdh & $\begin{array}{ll}\text { F: } & \text { GGAAAGCTGTGGCGTGAT } \\
\text { R: } & \text { AAGGTGGAAGAATGGGAGTT }\end{array}$ \\
\hline Aktl & $\begin{array}{ll}\mathrm{F}: & \text { CACGACCGCCTCTGCTTT } \\
\mathrm{R}: & \text { CACAGCCCGAAGTCCGTTA }\end{array}$ \\
\hline Ccl4 & $\begin{array}{ll}\mathrm{F}: & \text { TGCTGCTTCTCTTACACCTCC } \\
\mathrm{R}: & \text { TCATTCACATACTCATTGACCCA }\end{array}$ \\
\hline Cxcl3 & $\begin{array}{ll}\mathrm{F}: & \text { CAGTGCCTGAAGACCCTACCA } \\
\mathrm{R}: & \text { GATCGACTCGGACGTTATTTGA }\end{array}$ \\
\hline Stat3 & $\begin{array}{ll}\mathrm{F}: & \text { TTAACATTCTGGGCACGAACA } \\
\text { R: } & \text { TCAGTGACAATCAAGGAGGCA }\end{array}$ \\
\hline Tnfsfl1 & $\begin{array}{ll}\mathrm{F}: & \text { TACCTGGATAACCCTTGATGACC } \\
\mathrm{R}: & \text { TCTCCAGAAATCCCTACAACGG }\end{array}$ \\
\hline$C d 4$ & $\begin{array}{ll}\text { F: } & \text { TCAGCCCGACAGCAACACTT } \\
\text { R: } & \text { AGCACGACAGCCAGGAACAT }\end{array}$ \\
\hline$C s f 3 r$ & $\begin{array}{ll}\text { F: } & \text { GGTTCCATTCAAGACCCCAG } \\
\text { R: } & \text { TGTTTCCCTCAGGACCAGTAGA }\end{array}$ \\
\hline$H g f$ & $\begin{array}{ll}\mathrm{F}: & \text { TATTGCCCTATTTCCCGTTGT } \\
\mathrm{R}: & \text { CCATCCACCCTACTGTTGTTTG }\end{array}$ \\
\hline$I l 12 a$ & $\begin{array}{ll}\mathrm{F}: & \text { CAGCACTTCAGAGCCACAATC } \\
\mathrm{R}: & \text { GCCGCTGTGATTCAGAGACC }\end{array}$ \\
\hline Ill3 & $\begin{array}{ll}\mathrm{F}: & \text { AGTCCTGGCTCTCGCTTGC } \\
\mathrm{R}: & \text { TGTGTGATGTTGCTCAGCTCCT }\end{array}$ \\
\hline Illrl & $\begin{array}{ll}\text { F: } & \text { AAGTGGAATGGGTCGGAAAT } \\
\text { R: } & \text { AAGCAGATGAACGGATAGCG }\end{array}$ \\
\hline$I l 2$ & $\begin{array}{ll}\mathrm{F}: & \text { CACTTGGAAGACGCTGGAAAT } \\
\mathrm{R}: & \text { CACAGTTGCTGGCTCATCATC }\end{array}$ \\
\hline Il6st & $\begin{array}{ll}\text { F: } & \text { CGTGGCAGAAGTCCTCCTACA } \\
\text { R: } & \text { GGATCGCTTGAGCCTACATAAC }\end{array}$ \\
\hline$J a k 2$ & $\begin{array}{ll}\text { F: } & \text { AGAAGGGTGCCCAGACGA } \\
\text { R: } & \text { GGTTGACATTGTTGTTCCAGC }\end{array}$ \\
\hline Lifr & $\begin{array}{ll}\text { F: } & \text { CCGCCCTCTTATCCATCTTT } \\
\text { R: } & \text { ACCAGTCCCGTTATCCTTCC }\end{array}$ \\
\hline Mapk14 & $\begin{array}{ll}\text { F: } & \text { CTGTATTGTCAGGATTCTCGGA } \\
\text { R: } & \text { GCAGTGATGGGCTCTGGTTAG }\end{array}$ \\
\hline Mapkl & $\begin{array}{l}\text { F: CAGGAAAGCATTACCTTGACCAG } \\
\text { R: CAGAGCCTGTTCAACTTCAATCC }\end{array}$ \\
\hline Met & $\begin{array}{ll}\text { F: } & \text { GAAAATACCTCAACAGCGGCA } \\
\text { R: } & \text { AAAGATTTGGTCGGGTGGATT }\end{array}$ \\
\hline Mtor & $\begin{array}{ll}\text { F: } & \text { CCAACTACCTTCGGAACCTC } \\
\text { R: } & \text { CTTCACTTCAAACTCCACATACTC }\end{array}$ \\
\hline$N f k b l$ & $\begin{array}{ll}\text { F: ACTCAAGAACAGCAAGGCAGC } \\
\text { R: GGTGTCGTCCCATCGTAGGT }\end{array}$ \\
\hline Osm & $\begin{array}{ll}\text { F: } & \text { CAATGTTTACTGCATGGCTCG } \\
\text { R: } & \text { GGTCTGATTCTGTGGTCTCCCT }\end{array}$ \\
\hline Osmr & $\begin{array}{ll}\text { F: } & \text { ACTGTCCCAACCTTTAGTCATCA } \\
\text { R: } & \text { GCGTCATCTACCATAGCCCTTA }\end{array}$ \\
\hline Pias3 & $\begin{array}{ll}\text { F: } & \text { CTCСТTCCCAATACTCAGCG } \\
\text { R: } & \text { CAACCTTTATTGTAGGCGAGAA }\end{array}$ \\
\hline $\operatorname{Src}$ & $\begin{array}{ll}\text { F: } & \text { TGCTTCATACTGGGTGACGAG } \\
\text { R: } & \text { TGGGTAGAGTGGGTTGAGGTT }\end{array}$ \\
\hline
\end{tabular}

RT-qPCR, reverse transcription-quantitative polymerase chain reaction; $\mathrm{F}$, forward; $\mathrm{R}$, reverse.

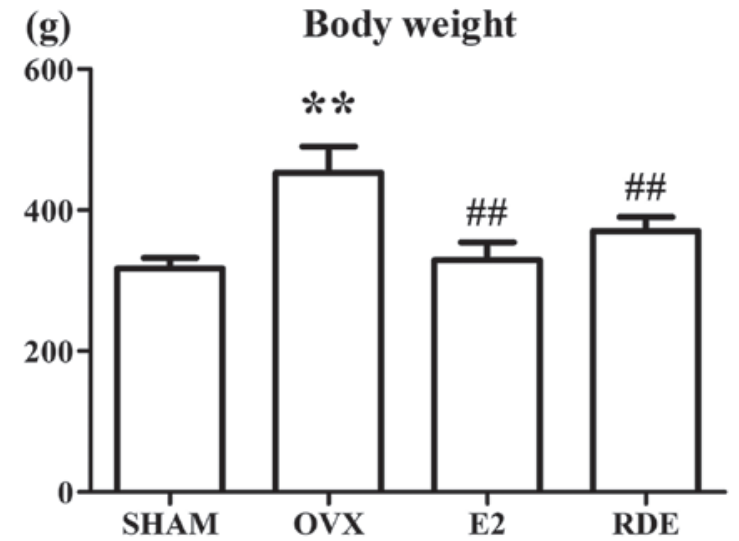

Figure 1. Effect of RDE on body weight after 12-week treatment; ${ }^{* *} \mathrm{P}<0.01$ vs. sham group; ${ }^{\# \#} \mathrm{P}<0.01$ vs. OVX group. RDE, Rhizoma Dioscoreae extract; OVX, ovariectomy; E2, 17 $\beta$-estradiol.

Confirmation by RT-qPCR. Alveolar bone from another 6 OVX and 6 RDE subject rats was used for RT-qPCR. The RNeasy mini kit (Qiagen, Valencia, CA, USA) was utilized to purify total extracted RNA and SuperScript First Strand Synthesis system (Invitrogen; Thermo Fisher Scientific, Carlsbad, CA, USA) was used to reversely transcribe $4 \mu \mathrm{g}$ RNA into cDNA. In an ABI-7500 Sequence Detection system (Applied Biosystems, Foster City, CA, USA), cDNA synthesized in the process of RT-qPCR was detected by SYBR-Green based on the manufacturer's instructions. The primers of the RT-qPCR analyses are listed in Table I. The RT-qPCR conditions in the present study were as follows: $10 \mathrm{sec}$ of initiation at $95^{\circ} \mathrm{C}, 5 \mathrm{sec}$ of thermal denaturation at $95^{\circ} \mathrm{C}$, and $34 \mathrm{sec}$ of annealing at $60^{\circ} \mathrm{C}$ for 40 cycles. The expressing quantities of glyceraldehyde-3-phosphate dehydrogenase (GAPDH) were used to normalize PCR results and $2^{-\Delta \Delta \mathrm{Cq}}$ was applied for data analysis. The purity of the amplified products was assessed by melting curves of each RT-qPCR assay containing negative control without templates.

Statistical analysis. Data are presented as mean \pm standardized difference and were statistically analyzed by SPSS 13.0 software (SPSS, Inc., Chicago, IL, USA). Analysis of variance and the least significant difference (LSD) test were used to determine inter-group differences in the parameters under evaluation. The normality of all data was proven by Kolmogorov-Smirnov tests, and a P-value of $<0.05$ was set as the threshold of statistical significance.

\section{Results}

Effect of RDE on body and uterine weight. The weight of sham rats was significantly lower compared with that of OVX rats (Fig. 1). Administration of E2 or RDE markedly inhibited weight gain induced by OVX during the 12 -week treatment.

Compared with the sham group, OVX was associated with marked atrophy of the uterus, which indicated a successful surgery. E2 injection markedly reduced the atrophy of uterine tissue compared with OVX rats, whereas administration of RDE exerted a mild uterotrophic effect (Fig. 2). 


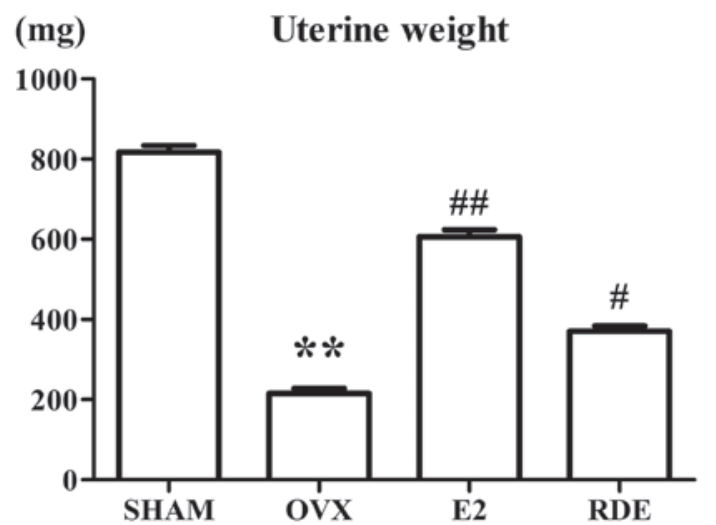

Figure 2. Effect of RDE on uterine weight after a 12-week treatment; ${ }^{* *} \mathrm{P}<0.01$ vs. sham group; ${ }^{*} \mathrm{P}<0.05$ vs. OVX group; ${ }^{\# \#} \mathrm{P}<0.01$ vs. OVX group. RDE, Rhizoma Dioscoreae extract; OVX, ovariectomy; E2, 17ß-estradiol.

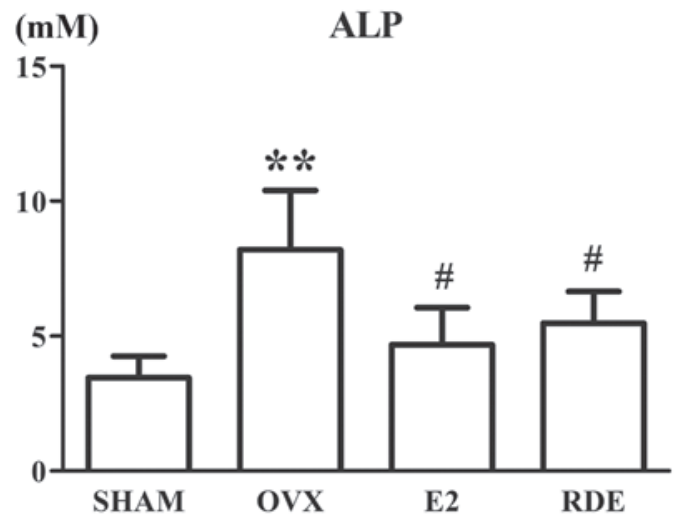

Figure 3. Effect of RDE on ALP in plasma after 12-week treatment; ${ }^{* * *} \mathrm{P}<0.01$ vs. sham group; ${ }^{*} \mathrm{P}<0.05$ vs. OVX group. RDE, Rhizoma Dioscoreae extract; OVX, ovariectomy; E2, 17ß-estradiol; ALP, alkaline phosphatase.

Effect of RDE on bone turnover biomarkers. The plasma concentrations of ALP and TRAP in different subject rats after a 12-week treatment are shown in Figs. 3 and 4. After 12 weeks, the ALP and TRAP levels in OVX rats were significantly higher compared with those in sham rats $(\mathrm{P}<0.01)$. Moreover, the plasma ALP and TRAP levels in RDE and E2 rats were significantly lower compared with those in OVX rats $(\mathrm{P}<0.01)$.

Effect of RDE on BMD and trabecular bone microarchitecture. It was revealed by analyzing morphological measurements that Tb.N, trabecular BV/TV and BMD were significantly decreased $(\mathrm{P}<0.01)$, while DA, SMI and Tb.Sp were significantly increased $(\mathrm{P}<0.01)$ in OVX rats compared with sham rats. Treatment with RDE or E2 relieved OVX-induced bone loss and limited the OVX-induced damage to alveolar bone trabeculae (Fig. 5 and Table II).

Effect of RDE on gene expression profile. It was revealed by IL-6/STAT3 signaling pathway PCR arrays that the expression of 24 genes from alveolar bone exhibited differences of $>3$-fold between OVX and RDE rats (Table III). Specifically, 2 genes were upregulated, while 22 were downregulated.

Confirmation of differential levels of gene expression by $R T-q P C R$. With the aim of confirming the differential gene

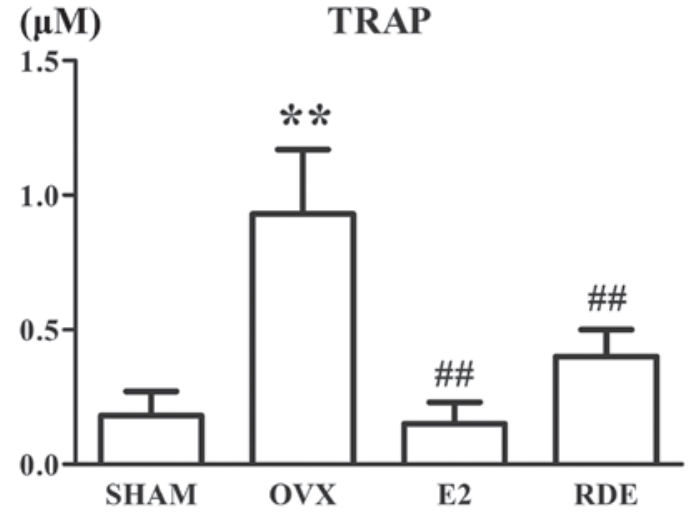

Figure 4. Effect of RDE on TRAP in plasma after 12-week treatment; ${ }^{* *} \mathrm{P}<0.01$ vs. sham group; ${ }^{\# \#} \mathrm{P}<0.05$ vs. OVX group. RDE, Rhizoma Dioscoreae extract; OVX, ovariectomy; E2, 17 $\beta$-estradiol; TRAP, tartrate-resistant acid phosphatase.

expression determined using IL-6/STAT3 signaling pathway PCR arrays, all 24 genes listed in Table III were verified using RT-qPCR and the results are presented in Fig. 6. Alveolar bone from 6 OVX and 6 RDE rats was used for the RT-qPCR assay. In the majority of the cases, the variations of genes in microarray analyses conformed to the RT-qPCR results, apart from 4 genes $(\mathrm{Cxcl} 3, \mathrm{Hgf}$, Il2 and $\mathrm{Ill2a}$ ). The role of the IL-6/STAT3 signaling pathway in the inhibitory effect of RDE on osteoporosis is schematically represented in Fig. 7.

\section{Discussion}

In our previous study, using bioinformatics and PCR or western blotting to confirm the expression of Stat3, it was demonstrated that RDE inhibited osteoporosis in OVX rats, an effect which was associated with the IL-6/oncostatin M (OSM)/STAT3 pathway via miRNA regulation (21). The putative miRNAs targets' gene prediction and pathway analysis are both bioinformatics methods, and the results occasionally do not fully reflect the true effects of RDE; however, the results raise the question whether RDE exerts an anti-osteopenic effect via another IL- 6 family cytokine pathway. The IL- 6 cytokine family includes 10 members: IL-6, IL-31, IL-27, IL-11, neuropoietin, cardiotrophin-like cytokine, cardiotrophin-1 (CT-1), ciliary neurotrophic factor (CNTF), OSM and leukemia inhibitory factor (LIF). All IL-6 family cytokines employ the transducing receptor $\beta$-subunit gp130 as part of a multimeric receptor complex (26). The present study aimed to determine the association between the IL- 6 family of cytokines pathway and the anti-osteopenic effect of RDE.

Our results demonstrated that treatment with RDE for 12 weeks prevented loss of uterine wet weight and body weight gain resulting from lack of estrogen in rats of the OVX group (Figs. 1 and 2). It was deduced that RDE exerts a mild estrogen-like effect, which may slow down OVX-induced uterine atrophy and weight gain.

Following ovary removal, OVX rats exhibited markedly reduced BMD, resulting from increasing alveolar bone metabolism, compared with the sham rats. By contrast, treatment with RDE or E2 increased the BMD of alveolar bone.

The significant and coincident increases in plasma ALP and TRAP verified mature female OVX rat as an appropriate 
Table II. Effect of RDE on BMD and trabecular bone microarchitecture.

\begin{tabular}{lcccc}
\hline & SHAM & OVX & E2 & RDE \\
\hline BMD $\left(\mathrm{g} / \mathrm{cm}^{3}\right)$ & $0.861 \pm 0.105$ & $0.344 \pm 0.017^{\mathrm{a}}$ & $0.601 \pm 0.004^{\mathrm{c}}$ & $0.499 \pm 0.089^{\mathrm{b}}$ \\
BV/TV $(\%)$ & $27.708 \pm 4.371$ & $7.839 \pm 1.364^{\mathrm{a}}$ & $17.631 \pm 1.765^{\mathrm{c}}$ & $13.886 \pm 2.888^{\mathrm{b}}$ \\
Tb.Th $(\mu \mathrm{m})$ & $24.989 \pm 0.165$ & $24.473 \pm 0.173$ & $24.568 \pm 0.518$ & $24.491 \pm 0.595$ \\
Tb.Sp $(\mu \mathrm{m})$ & $48.646 \pm 12.107$ & $93.224 \pm 7.745^{\mathrm{a}}$ & $60.646 \pm 12.360^{\mathrm{c}}$ & $66.763 \pm 12.060^{\mathrm{b}}$ \\
Tb.N $(1 / \mathrm{mm})$ & $0.011 \pm 0.002$ & $0.003 \pm 0.000^{\mathrm{a}}$ & $0.007 \pm 0.001^{\mathrm{c}}$ & $0.006 \pm 0.001^{\mathrm{b}}$ \\
SMI & $1.206 \pm 0.118$ & $2.090 \pm 0.122^{\mathrm{a}}$ & $1.523 \pm 0.115^{\mathrm{c}}$ & $1.680 \pm 0.147^{\mathrm{c}}$ \\
DA & $1.330 \pm 0.150$ & $1.834 \pm 0.095^{\mathrm{a}}$ & $1.455 \pm 0.267^{\mathrm{b}}$ & $1.584 \pm 0.111$ \\
\hline
\end{tabular}

Values are presented as means \pm standardized difference ( $\mathrm{n}=12$ in each group). ${ }^{\mathrm{a}} \mathrm{P}<0.01 \mathrm{vs}$. SHAM group; ${ }^{\mathrm{b}} \mathrm{P}<0.05 \mathrm{vs}$. OVX group; ${ }^{\mathrm{c}} \mathrm{P}<0.01 \mathrm{vs}$. OVX group. RDE, Rhizoma Dioscoreae extract; BMD, bone mineral density; OVX, ovariectomy; E2, 17 $\beta$-estradiol; BV/TV, bone volume fraction; Tb.Th, trabecular thickness; Tb.Sp, trabecular separation; Tb.N, trabecular number; SMI, structure model index; DA, degree of anisotropy.

Table III. Differential expression of genes ( $\geq 2$-fold) in alveolar bone from 6 RDE and 6 OVX rats.

\begin{tabular}{|c|c|c|}
\hline Symbol & $\mathrm{P}$-value & Fold-change \\
\hline Aktl & 0.000264 & -3.09 \\
\hline Ccl4 & 0.001408 & -3.26 \\
\hline$C d 4$ & 0.000581 & -2.34 \\
\hline$C s f 3 r$ & 0.001814 & -4.45 \\
\hline Cxcl3 & 0.023671 & -11.72 \\
\hline$H g f$ & 0.004032 & -3.30 \\
\hline$I l 12 a$ & 0.027687 & -2.87 \\
\hline$I l 13$ & 0.019136 & 2.06 \\
\hline Illrl & 0.000045 & -3.85 \\
\hline$I l 2$ & 0.027486 & 5.58 \\
\hline Il6st & 0.005906 & -2.88 \\
\hline Jak2 & 0.000009 & -2.31 \\
\hline Lifr & 0.009917 & -3.25 \\
\hline Mapk14 & 0.000994 & -2.81 \\
\hline Mapkl & 0.040108 & -1.64 \\
\hline Met & 0.000938 & -5.16 \\
\hline Mtor & 0.010758 & -2.03 \\
\hline$N f k b 1$ & 0.000017 & -3.05 \\
\hline Osm & 0.040752 & -4.52 \\
\hline Osmr & 0.016804 & -2.01 \\
\hline Pias3 & 0.007814 & -2.75 \\
\hline Src & 0.030115 & -2.34 \\
\hline Stat3 & 0.004448 & -2.57 \\
\hline $\operatorname{Tnfsf11}$ & 0.007872 & -6.58 \\
\hline
\end{tabular}

RDE, Rhizoma Dioscoreae extract; OVX, ovariectomy.

and reliable animal model for studies on early postmenopausal osteoporosis characterized by high-turnover bone loss. Treating rats with RDE or E2 for 12 weeks inhibited the excessive bone metabolism, as shown by significantly decreased ALP and TRAP levels (Figs. 3 and 4).

From analyses of 3-D bone microarchitecture by micro-CT, it was indicated that the loss of alveolar bone among rats treated with E2 or RDE was less severe compared with that in
OVX rats, which was inferred through significant variations in SMI, Tb.Sp, Tb.N and BV/TV. The inhibition of alveolar bone loss with E2 was superior to that of RDE (Table II and Fig. 5).

The results of bone metabolic biomarkers, micro-CT and BMD demonstrated that RDE significantly inhibited alveolar bone loss.

To investigate the association between the IL-6/STAT3 signaling pathway and the anti-osteopenic effect of RDE, the differential expression of genes by was screened using IL-6/STAT3 signaling pathway array. A total of 20 genes (19 downregulated and 1 upregulated) from alveolar bone were successfully identified and validated; their expression profiles differed significantly among RDE rats compared with the OVX group. The IL-6/STAT3 signaling pathway was downregulated following RDE treatment (Fig. 7).

In the IL-6 family cytokine signaling cascade, oligomerization of receptor subunits induced by a specific ligand may activate janus protein-tyrosine kinases (JAKs), which further activate the MAPKs or the STATs (mainly STAT1 and STAT3). Another signaling cascade activated by cytokines of the IL-6 family is the phosphoinositide-3-kinase/AKT $(27,28)$. Receptor complexes that are capable of signaling after being activated by cytokines of the IL-6 family may provide specificity in a given signal transduction pathway. For example, OSM is considered to be a unique gp130-binding cytokine, as it binds first to gp130, and then develops a signaling complex with either the OSM receptor (OSMR) or the LIF receptor (LIFR), which are both capable of intracellular signaling $(29,30)$.

Cytokines of the IL- 6 family exert a major effect on osseous remodeling through osteoclasts or osteoblasts, despite seemingly functioning as a double-edged sword. These cytokines maintain bone generation while driving bone absorption induced by a variety of osteolytic factors. This dual effect is apparent in two main signaling pathways (STAT and MAPK signaling) bidirectionally affecting osteocytes (19).

Certain members of the IL-6 family (OSM, CNTF, LIF, IL-11 and IL-6) may promote bone formation. This effect was mainly reflected in three aspects: First, these cytokines promote the differentiation of osteoblast precursors or maturing osteoblasts isolated from the bone marrow or calvaria. During this process, STAT3 must be activated $(31,32)$. Thus, the expression of osteoblastic biomarkers, such as bone sialoprotein, osteocalcin or ALP, was increased, while extracellular matrix 

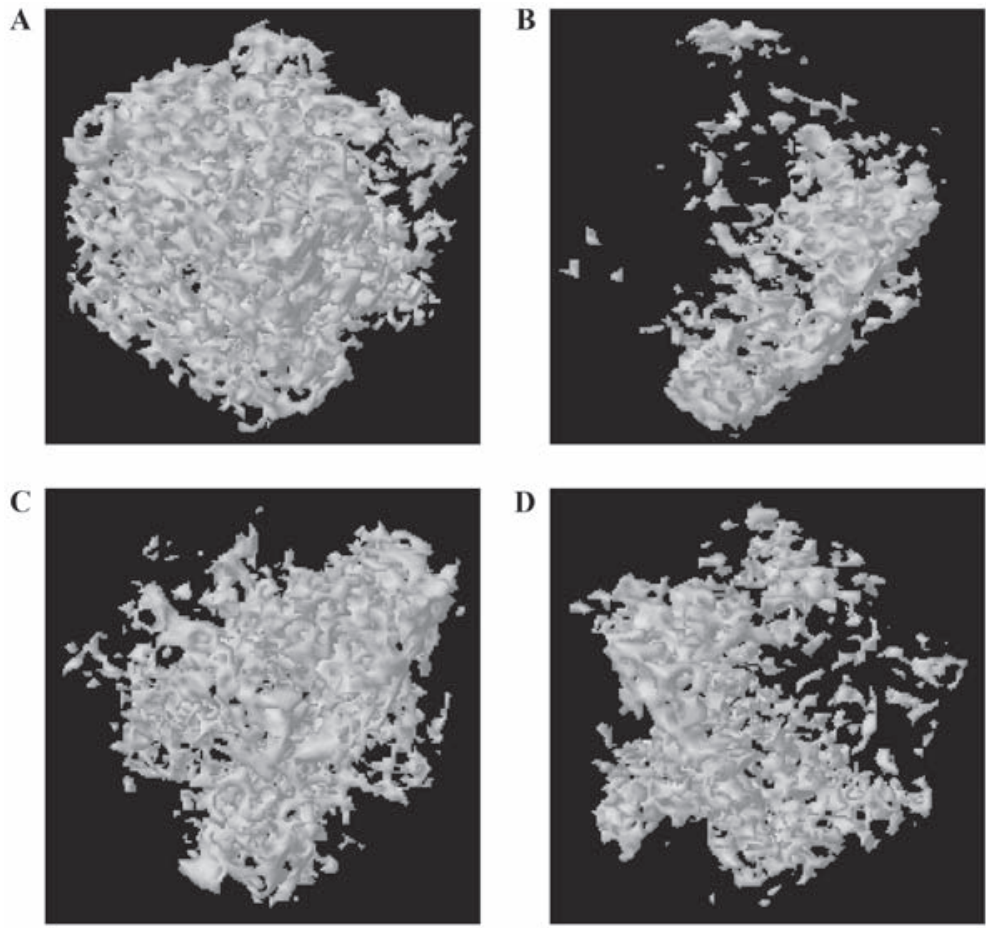

Figure 5. Representation of 3-D architecture of alveolar bone beneath the lowest point of the first molar crown in the (A) sham, (B) OVX, (C) E2 and (D) RDE groups. RDE, Rhizoma Dioscoreae extract; OVX, ovariectomy; E2, 17ß-estradiol.
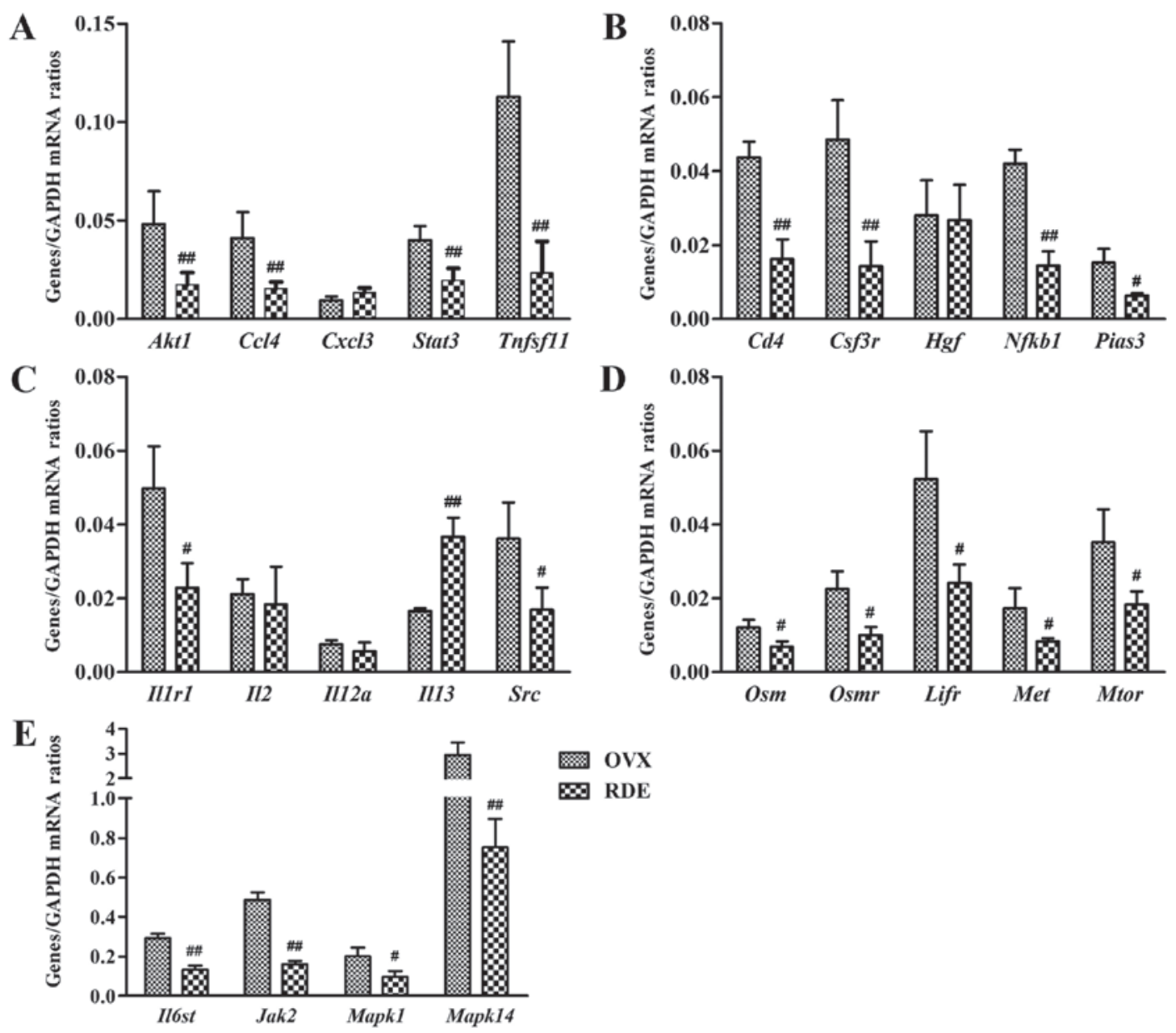

Figure 6. Validation of 24 differential expression genes identified by microarray and IPA in a replicated experiment by RT-qPCR. (A-E) Effect of RDE on the expressions of Akt1, Ccl4, Cd4, Csf3r, Cxcl3, Hgf, Ill2a, Ill3, Illr1, Il2, Il6st, Jak2, Lifr, Mapk14, Mapk1, Met, Mtor, Nfkb1, Osm, Osmr, Pias3, Src, Stat3 and Tnfsf11. ${ }^{\#} \mathrm{P}<0.05$ and ${ }^{\# \# /} \mathrm{P}<0.01$ compared with the OVX group. Alveolar bone prepared from 6 RDE and 6 OVX rats was used. IPA, Ingenuity Pathway Analysis; RDE, Rhizoma Dioscoreae extract; OVX, ovariectomy; RT-qPCR, reverse transcription-quantitative polymerase chain reaction. 


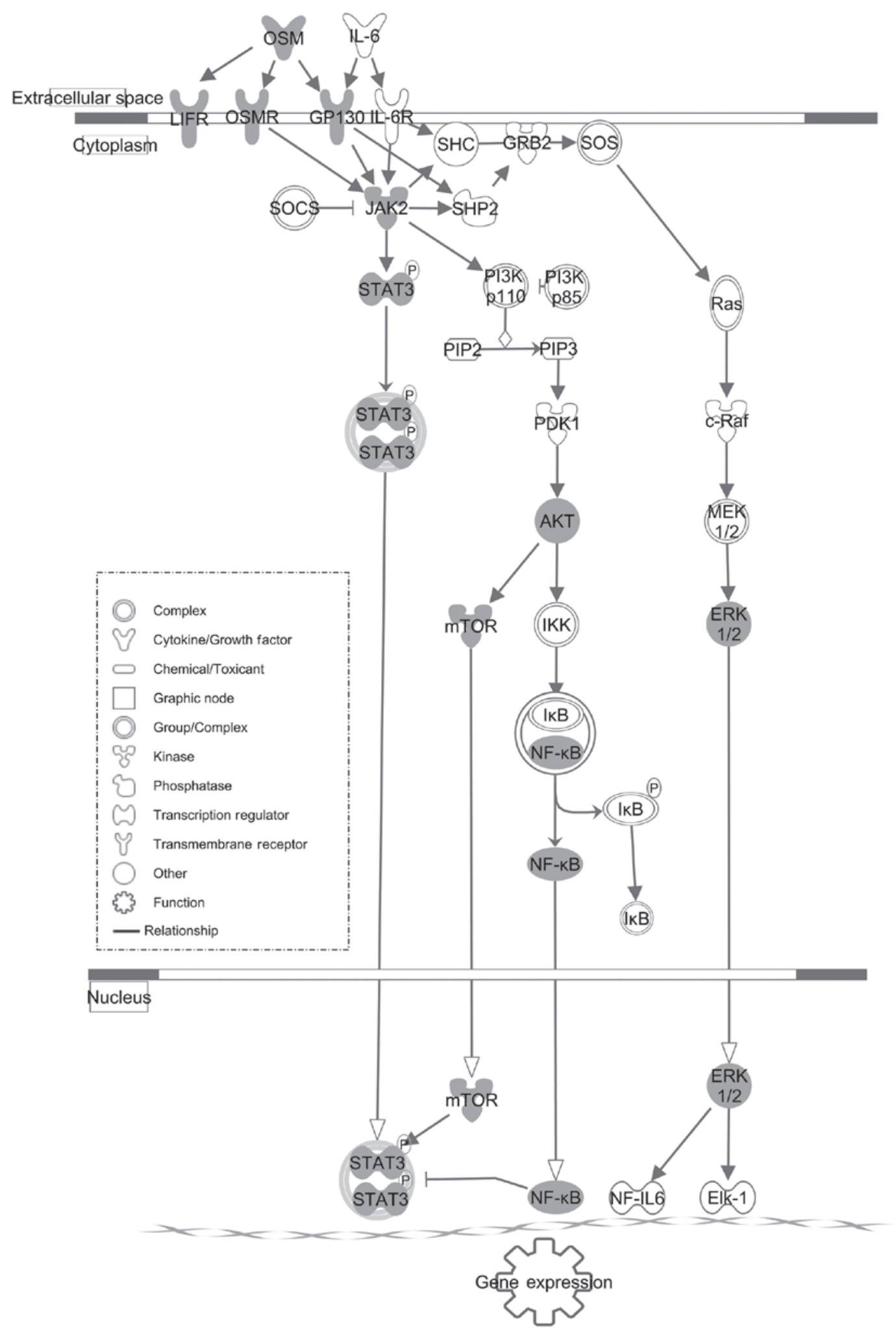

Figure 7. Schematic diagram illustrating the role of IL-6/STAT3 signaling pathway in an anti-osteopenic effect of RDE. The downregulated genes appear in gray. The genes that are not specified but are incorporated into the network through association appear in white. RDE, Rhizoma Dioscoreae extract; IL-6, interleukin-6; STAT3, signal transducer and activator of transcription 3.

mineralization and bone nodule formation were enhanced. Second, the biomarkers inhibit certain osteoblasts from proliferating via activating gp130/STAT3 and increasing the expression of $\mathrm{p} 21^{\mathrm{WAF} 1}$, which is a cell cycle inhibitor and is also required for cytokines of the IL-6 family to induce ALP (33). Finally, these cytokines may protect osteoblasts from apoptosis caused by tumor necrosis factor- $\alpha$ (TNF- $\alpha$ ) or serum depletion (34).

As regards bone absorption, cytokines of the IL- 6 family promote osteoclast differentiation and bone absorption by accelerating the interactions between osteoclasts and osteoblasts. In co-cultures of osteoclast precursors and stromal cells 
or osteoblasts, certain IL-6-type cytokines (OSM, CNTF, LIF, IL-11 and IL-6) are able to promote osteoclast differentiation and bone absorption. In fact, these cytokines induce production of various osteoblastic downstream effectors that, in turn, promote osteoclast differentiation or activity, such as IL-1, receptor activator of nuclear factor- $\kappa \mathrm{B}(\mathrm{NF}-\kappa \mathrm{B})$ ligand (RANKL), prostaglandin E2 and PTH-related protein. Recent studies implicated activation of IL-6-type cytokines/STAT3 signaling as the pivotal event in the induction of RANKL in osteoblasts, which leads to pro-resorption action of osteoclasts $(35,36)$.

It was observed that the gene expression of certain signaling molecules (Osm, Osmr, Lifr, Il6st, Jak2, Stat3, Mtor, Nfkb1 and Mapk1) in the IL-6/STAT3 pathway were downregulated following RDE treatment (Fig. 7), which subsequently inhibited IL-6/STAT3 signaling. The RT-qPCR analysis of Osm, Osmr, Lifr, Il6st, Jak2, Stat3, Mtor, Nfkbl and Mapkl expression (Fig. 6) proved that RDE effectively reduced excessive alveolar bone formation and bone absorption synchronously caused by attenuated canonical IL-6/STAT3 signaling following OVX.

Further studies are required for RDE to be developed into a novel promising drug for the prevention or treatment of alveolar bone loss in postmenopausal women.

In conclusion, RDE was effective in inhibiting rat alveolar bone loss caused by OVX, by simultaneously inhibiting bone formation as well as bone resorption through regulation of the IL-6/STAT3 signaling pathway. The present study verified that RDE may be used as an oral agent for the treatment of alveolar osteopenia in postmenopausal women.

\section{Acknowledgements}

The present study was supported by the National Natural Science Foundation of China (grant no. 81473450) and the Fundamental Research Funds for the Beijing Administration of Traditional Chinese Medicine (grant no. JJ2015-54).

\section{References}

1. Gallagher JC and Levine JP: Preventing osteoporosis in symptomatic postmenopausal women. Menopause 18: 109-118, 2011.

2. Sultan N and Rao J: Association between periodontal disease and bone mineral density in postmenopausal women: A cross sectional study. Med Oral Patol Oral Cir Bucal 16: e440-e447, 2011.

3. Lee BD and White SC: Age and trabecular features of alveolar bone associated with osteoporosis. Oral Surg Oral Med Oral Pathol Oral Radiol Endod 100: 92-98, 2005.

4. Streckfus CF, Johnson RB, Nick T, Tsao A and Tucci M Comparison of alveolar bone loss, alveolar bone density and second metacarpal bone density, salivary and gingival crevicular fluid interleukin- 6 concentrations in healthy premenopausal and postmenopausal women on estrogen therapy. J Gerontol A Biol Sci Med Sci 52: M343-M351, 1997.

5. Tezal M, Wactawski-Wende J, Grossi SG, Dmochowski J and Genco RJ: Periodontal disease and the incidence of tooth loss in postmenopausal women. J Periodontol 76: 1123-1128, 2005.

6. Civitelli R,Pilgram TK,DotsonM,Muckerman J,Lewandowski N Armamento-Villareal R, Yokoyama-Crothers N, Kardaris EE, Hauser J, Cohen S, et al: Alveolar and postcranial bone density in postmenopausal women receiving hormone/estrogen replacement therapy: A randomized, double-blind, placebo-controlled trial. Arch Intern Med 162: 1409-1415, 2002.

7. Liu J, Cao Z and Li C: Intermittent PTH administration: A novel therapy method for periodontitis-associated alveolar bone loss. Med Hypotheses 72: 294-296, 2009.

8. Palomo L, Bissada NF and Liu J: Periodontal assessment of postmenopausal women receiving risedronate. Menopause 12: 685-690, 2005
9. Strom BL, Schinnar R, Weber AL, Bunin G, Berlin JA, Baumgarten M, DeMichele A, Rubin SC, Berlin M, Troxel AB, et al: Case-control study of postmenopausal hormone replacement therapy and endometrial cancer. Am J Epidemiol 164: 775-786, 2006.

10. Rossing MA, Cushing-Haugen KL, Wicklund KG, Doherty JA and Weiss NS: Menopausal hormone therapy and risk of epithelial ovarian cancer. Cancer Epidemiol Biomarkers Prev 16: 2548-2556, 2007.

11. Rizzoli R, Reginster JY, Boonen S, Bréart G, Diez-Perez A, Felsenberg D, Kaufman JM, Kanis JA and Cooper C: Adverse reactions and drug-drug interactions in the management of women with postmenopausal osteoporosis. Calcif Tissue Int 89: 91-104, 2011.

12. Woo SB, Hellstein JW and Kalmar JR: Narrative [corrected] review: Bisphosphonates and osteonecrosis of the jaws. Ann Intern Med 144: 753-761, 2006.

13. Clemett D and Spencer CM: Raloxifene: A review of its use in postmenopausal osteoporosis. Drugs 60: 379-411, 2000

14. Sugimoto H1, Watanabe K, Toyama T, Takahashi SS, Sugiyama S, Lee MC and Hamada N: Inhibitory effects of French pine bark extract, Pycnogenol ${ }^{\circledR}$, on alveolar bone resorption and on the osteoclast differentiation. Phytother Res 29: 251-259, 2015.

15. Sağlam M, Köseoğlu S, Hatipoğlu M, Esen HH and Köksal E: Effect of sumac extract on serum oxidative status, RANKL/OPG system and alveolar bone loss in experimental periodontitis in rats. J Appl Oral Sci 23: 33-41, 2015.

16. Guimarães MV,Melo IM, Adriano Araújo VM, Tenazoa Wong DV, Roriz Fonteles CS, Moreira Leal LK, Ribeiro RA and Lima V: Dry extract of Matricaria recutita L. (Chamomile) prevents ligature-induced alveolar bone resorption in rats via inhibition of tumor necrosis factor- $\alpha$ and interleukin-1 $\beta$. J Periodontol 87: 706-715, 2016.

17. Feng XF, Huang LQ, Ge XG, Yang LJ and Yang JY: Textual research on origin and development of genuine medicinal herbs of Shanyao. Zhongguo Zhong Yao Za Zhi 33: 859-862, 2008 (In Chinese).

18. Zhang Z, Xiang L, Bai D, Wang W, Li Y, Pan J, Liu H, Wang S, Xiao GG and Ju D: The protective effect of Rhizoma Dioscoreae extract against alveolar bone loss in ovariectomized rats via regulating Wnt and p38 MAPK signaling. Nutrients 6: 5853-5870, 2014.

19. Blanchard F, Duplomb L, Baud' huin M and Brounais B: The dual role of IL-6-type cytokines on bone remodeling and bone tumors. Cytokine Growth Factor Rev 20: 19-28, 2009.

20. Sims NA and Walsh NC: GP130 cytokines and bone remodelling in health and disease. BMB Rep 43: 513-523, 2010

21. Zhang Z, Song C, Zhang F, Xiang L, Chen Y, Li Y, Pan J, Liu H, Xiao GG and Ju D: Rhizoma Dioscoreae extract protects against alveolar bone loss in ovariectomized rats via microRNAs regulation. Nutrients 7: 1333-1351, 2015.

22. Zhang Z, Xiang L, Bai D, Fu X, Wang W, Li Y, Liu H, Pan J, Li Y, Xiao GG, et al: Treatment with Rhizoma Dioscoreae extract has protective effect on osteopenia in ovariectomized rats. ScientificWorldJournal 2014: 645975, 2014.

23. Hidaka S, Okamoto Y, Yamada Y, Kon Y and Kimura T: A Japanese herbal medicine, Chujo-to, has a beneficial effect on osteoporosis in rats. Phytother Res 13: 14-19, 1999.

24. Yang J, Pham SM and Crabbe DL: High-resolution micro-CT evaluation of mid- to long-term effects of estrogen deficiency on rat trabecular bone. Acad Radiol 10: 1153-1158, 2003.

25. Bouxsein ML, Boyd SK, Christiansen BA, Guldberg RE, Jepsen KJ and Müller R: Guidelines for assessment of bone microstructure in rodents using micro-computed tomography. J Bone Miner Res 25: 1468-1486, 2010.

26. Garbers C, Hermanns HM, Schaper F, Müller-Newen G, Grötzinger J, Rose-John S and Scheller J: Plasticity and cross-talk of interleukin 6-type cytokines. Cytokine Growth Factor Rev 23: 85-97, 2012.

27. Grant SL and Begley CG: The oncostatin M signalling pathway: Reversing the neoplastic phenotype? Mol Med Today 5: 406-412, 1999.

28. Heinrich PC,BehrmannI,HaanS,Hermanns HM,Müller-Newen G and Schaper F: Principles of interleukin (IL)-6-type cytokine signalling and its regulation. Biochem J 374: 1-20, 2003.

29. Mosley B, De Imus C, Friend D, Boiani N, Thoma B, Park LS and Cosman D: Dual oncostatin M (OSM) receptors. Cloning and characterization of an alternative signaling subunit conferring OSM-specific receptor activation. J Biol Chem 271: 32635-32643, 1996.

30. Lindberg RA, Juan TS, Welcher AA, Sun Y, Cupples R, Guthrie B and Fletcher FA: Cloning and characterization of a specific receptor for mouse oncostatin M. Mol Cell Biol 18: 3357-3367, 1998. 
31. Bellido T, Borba VZ, Roberson P and Manolagas SC: Activation of the Janus kinase/STAT (signal transducer and activator of transcription) signal transduction pathway by interleukin-6-type cytokines promotes osteoblast differentiation. Endocrinology 138: 3666-3676, 1997.

32. Itoh S, Udagawa N, Takahashi N, Yoshitake F, Narita H, Ebisu S and Ishihara K: A critical role for interleukin-6 family-mediated Stat 3 activation in osteoblast differentiation and bone formation. Bone 39: 505-512, 2006.

33. Bellido T, O' Brien CA, Roberson PK and Manolagas SC: Transcriptional activation of the p21(WAF1, CIP1, SDI1) gene by interleukin- 6 type cytokines. A prerequisite for their pro-differentiating and anti-apoptotic effects on human osteoblastic cells. J Biol Chem 273: 21137-21144, 1998.

34. Jilka RL, Weinstein RS, Bellido T, Parfitt AM and Manolagas SC: Osteoblast programmed cell death (apoptosis): modulation by growth factors and cytokines. J Bone Miner Res 13: 793-802, 1998.
35. Palmqvist P, Persson E, Conaway HH and Lerner UH: IL-6, leukemia inhibitory factor, and oncostatin M stimulate bone resorption and regulate the expression of receptor activator of NF-kappa B ligand, osteoprotegerin, and receptor activator of NF-kappa B in mouse calvariae. J Immunol 169: 3353-3362, 2002.

36. Kim S, Yamazaki M, Shevde NK and Pike JW: Transcriptional control of receptor activator of nuclear factor-kappaB ligand by the protein kinase A activator forskolin and the transmembrane glycoprotein 130 -activating cytokine, oncostatin $\mathrm{M}$, is exerted through multiple distal enhancers. Mol Endocrinol 21: 197-214, 2007. 